\title{
VALUE OF SENTINEL NODE BIOPSY IN ANAL CANCER PATIENTS
}

\author{
Nicolae BACALBASA ${ }^{1,2,3 凶}$, Irina BALESCU ${ }^{4}$, Cristian BALALAU ${ }^{2,5}$, Mihaela VILCU ${ }^{2,3}$, Iulian \\ BREZEAN ${ }^{2,3}$
}

${ }^{1}$ Center of Excellence in Translational Medicine, Fundeni Clinical Institute, Bucharest, Romania

2 "Carol Davila“ University of Medicine and Pharmacy, Bucharest, Romania

3 „Ion Cantacuzino“ Clinical Hospital, Bucharest, Romania

${ }^{4}$ Ponderas Academic Hospital, Bucharest, Romania

5 „Sf. Pantelimon“ Clinical Hospital, Bucharest, Romania

\section{Abstract}

Anal cancer is a rare digestive malignancy with high propensity to spread via lymphatic route, leading to the apparition of metastases at the level of superficial inguinal and perirectal stations. However, prophylactic irradiation of all these fields is not recommended due to the inacceptable high risks of complications when compared to the rates of metastases. Therefore, sentinel lymph node biopsy has been routinely associated in order to determine which cases present lymphatic metastases and could benefit most after irradiation. This is a literature review of the largest studies conducted on the subject of sentinel lymph node biopsy in anal cancer patients.

Keywords: anal cancer, sentinel node biopsy, metastasis, irradiation.

\section{RÉSUMÉ}

Valeur de la biopsie du ganglion sentinelle chez les patients atteints d'un cancer anal

Le cancer anal est une tumeur maligne digestive rare avec une forte propension à se propager par voie lymphatique, conduisant à l'apparition de métastases au niveau des stations superficielles inguinales et péri-rectales. Cependant, une irradiation prophylactique de tous ces champs n'est pas recommandée en raison de complications par rapport aux taux de métastases. Par conséquent, la biopsie du ganglion sentinelle a été systématiquement associée afin de déterminer les cas présentant des métastases lymphatiques et pouvant en tirer le plus grand bénéfice après l'irradiation. Il s'agit d'une revue de la littérature des plus grandes études menées sur le sujet de la biopsie du ganglion sentinelle chez des patients atteints d'un cancer de l'anus.

Mots-clés: cancer de l'anus, biopsie du ganglion sentinelle, métastases, irradiation. 


\section{INTRODUCTION}

Anal cancer represents a rare digestive malignancy which usually metastasizes via the lymphatic route. However, the estimated incidence of this malignancy has encountered a permanent increase in the last decades, especially due to the association with Human Papilloma Virus infection ${ }^{1,2}$. The presence of lymph node metastases represents one of the most important prognostic factors being associated with higher rates of recurrence and decreased overall survival rates ${ }^{3,4}$. Therefore, the status of the lymph nodes plays a crucial role in order to establish the therapeutic protocol; the most commonly involved lymph node stations are represented by the superficial inguinal nodes and, in certain cases, the pelvic and mesenteric lymph nodes ${ }^{5,6}$. The exact location of the metastatic nodes is essential in order to determine which should be the areas included in the radiation field ${ }^{7-9}$. However, an interesting observation is related to the fact that only the presence of inguinal lymph node metastases influences the prognosis of anal cancer patients; Gerard et al report a significantly worsened prognosis in patients presenting larger than $2 \mathrm{~cm}$ inguinal lymph nodes, while the association of synchronous perirectal adenopathies does not seem to influence the long-term outcomes ${ }^{3}$. Moreover, it seems that up to $5 \%$ of cases will develop bilateral synchronous or metachronous lymph node metastases; in consequence, a proper evaluation of the inguinal lymph node stations is mandatory in order to provide an adequate staging and an adequate therapeutic strategy to increase the overall survival ${ }^{3}$.

\section{Therapeutic StRategies IN ANAL CANCER PATIENTS}

In the last decades, the first intention treatment in anal cancer has been represented by chemo-irradiation, while in cases developing local complications or recurrences, surgery might be taken in consideration. However, performing a surgical procedure with curative intent in such a patient is associated with higher perioperative morbidity rates, and therefore, is reserved for cases that failed after all the other attempts of conservative treatment ${ }^{10,11}$. Since 1974, when Nigro proposed a new therapeutic protocol based on irradiation with concurrent sensitization chemotherapy, this became the standard of care in anal cancer patients ${ }^{12}$. The radiation field used in such cases usually comprises perirectal area but does not routinely include the inguinal lymphatic stations. However, the routine inclusion of the groin area in the field of irradiation is controversial due to the fact that less than one quart of the patients will develop lymph node metastases at this level ${ }^{9}$. Moreover, association of groin irradiation can induce significant complications such as skin necrosis, lymphedema or blood vessel damage leading to the development of a chronic obstructive disease ${ }^{3,13}$. In cases with anal cancer traditionally two therapeutic strategies have been proposed: the first one consisted of routine prophylactic groin irradiation, and was associated in several papers with better results when compared to cases submitted to routine extended lymph node dissection. The second one consisted of a follow-up protocol and irradiation of cases who are diagnosed at a certain point of their evolution with metachronous inguinal metastases ${ }^{3,14}$.

Classically, it has been considered that clinical examination of the inguinal area by palpation, in association with inguinal ultrasound and fine needle aspiration of the suspected lymph nodes, should provide an efficient evaluation of these lymphatic stations. However, up to $44 \%$ of cases presenting smaller than $5 \mathrm{~mm}$ metastatic deposits might skip from this evaluation ${ }^{4}$. In this respect, most authors agree that the histopathological status of the lymph node stations by performing a sentinel node detection technique should be investigated before establishing the extent of the irradiation field.

\section{Sentinel Lymph NODE BIOPSY IN ANAL CANCER PATIENTS}

Due to the relatively low number of patients diagnosed with anal cancer, the method of sentinel lymph node biopsy couldn't be studied on large number of cases; however, the method seems to be safe and effective in order to determine which are the cases who will benefit from groin irradiation ${ }^{10,11}$. The technique was proposed for the first time in anal cancer patients by John Spratt in 2000 in order to avoid performing prophylactic groin lymph node dissection ${ }^{15}$.

The identification of sentinel node biopsy in anal cancer patients can be performed after injecting radiocolloid products such as Technetium 99 and/or after injecting detection dye. Due to the localization of the tumour, the administration of the tracer is very facile to be performed, under direct visual examination; this fact can maybe explain why in certain studies the detection rate of the sentinel node reaches $100 \%{ }^{16-18}$.

In cases in which radiocolloid injection is the option of choice, the protocol consists of a preoperative administration of Technetium 99 in the peritumoral area followed by pelvic lymphoscintigraphy and surgery one day later. In cases in which a dual method is desired, at the time of surgery, peritumoral injection of blue dye (such as patent blue) can be performed in the same points as the radiotracer; further on, the 
surgery consists of performing of a small skin incision at the level where the gamma probe detects a peak of radioactive signal and searching the radioactive and coloured lymph nodes ${ }^{16}$. Similarly to breast cancer or malignant melanoma, it seems that the dual method is associated with higher rates of sentinel lymph node detection in anal cancer too ${ }^{19,20}$. Moreover, the dual method is associated with a lower time to detection of the sentinel nodes and plays an essential role in cases presenting midline lesions and in which bilateral sentinel lymph node detection is expected ${ }^{16-20}$.

As for the cases presenting large palpable groin masses or other evident signs of nodal involvement, the method of sentinel lymph node detection is not reliable due to the presence of synchronous obstructions of the normal lymphatic drainage channels; therefore, in such cases the method of sentinel node biopsy should not be performed, the presence of palpable lymph nodes being considered as a formal contraindication $^{21,22}$.

Contrarily to other malignant diseases in which the concept of sentinel lymph node dissection has been widely implemented, in patients with anal cancer the rates of sensitivity and specificity of the method are very difficult to be established due to the fact that most of the studies do not complete the procedure with an extended groin lymph node dissection. This fact is also related to the extremely high rates of postprocedural complications if extended lymph node dissection is carried out; so, the status of the non-sentinel lymph nodes is not known and, in consequence, the sensitivity, and the specificity of the method cannot be established. The only value which can be calculated remains the negative predictive value; however, for this determination, the patients should be closely supervised for a long period of time in order to detect the development of metachronous lymph node metastases ${ }^{23,24}$. An interesting study which was conducted on this subject was published by Bobin et al in $2003^{18}$. The authors included in this paper 35 anal cancer patients submitted to sentinel node biopsy with a reported detection rate of $100 \%$. Among the 35 cases, there were nine nodes presenting lymph node metastases. After a median follow-up period of 18 months, none of the cases with negative sentinel lymph node did experience inguinal lymph node metastases ${ }^{18}$.

In a review study conducted by Noorani et al and published in 2013, the authors included 17 studies and 270 patients submitted to sentinel lymph node detection ${ }^{25}$. Among these patients, the detection rate ranged from $47 \%$ to $100 \%$, while the per cent of cases presenting nodal metastases ranged from 0 to $44 \%$; the authors underlined the fact that using radiocolloid alone was associated with poorer rates of sentinel node detection, while using a dual method increased the accuracy of the method; as for the rate of nodal metastases, the authors demonstrated that a detection rate of 0 was rather correlated with a small number of cases included in the respective study. As mentioned before, the false negative rate could not be established due to the fact that sentinel node biopsy was not further associated with lymph node dissection; however, this rate was estimated by supervising the patients during a longer period of time and was represented by the per cent of the cases with negative sentinel nodes who did not undergo adjuvant radiation therapy and who developed at a certain moment inguinal metastases. According to this definition, the false negative rate ranged between 0 and $18.75 \%{ }^{25}$.

One of the largest studies conducted on the theme of sentinel node detection in anal cancer patients was conducted by Mistrangelo et al and was published in the British Journal of Cancer in 201326. The study included 63 patients diagnosed with anal cancer and submitted to sentinel node detection by radioisotope injection at four cardinal points around the tumour, one day before surgery; in $59.7 \%$ of cases bilateral migration of the radiocolloid was encountered. Overall the detection rate was $98.4 \%$, while the median number of retrieved nodes was 4 . Among the analysed specimens, metastases were encountered in 13 cases, two of them presenting bilateral tumoral deposits. Moreover, the authors compared the results after sentinel lymph node biopsy with those provided after performing a PET/CT and demonstrated that the imagistic studies were able to detect the presence of the inguinal metastatic disease in only eight cases. After a median follow-up period of 35 months, none of the patients presenting negative sentinel nodes developed metachronous metastases, although adjuvant radiation therapy was not administered ${ }^{26}$.

\section{Tailoring the subsequent treatment according to the sentinel lymph node status}

Once the status of the sentinel node is established, the further treatment consisting of groin irradiation can be decided. In this way the rates of unnecessary irradiation are reduced as much as possible. Since 2001, sentinel lymph node biopsy has been routinely applied in anal cancer patients in order to plan the groin radiotherapy. Moreover, the method seems to help detecting occult inguinal disease and to select the cases in which radiation is needed ${ }^{27,28}$. In this respect, De Nardi et al proposed that node positive patients should be further submitted to groin irradiation while groin negative cases should only be carefully supervised; using this protocol, a significant decrease of the radiation related morbidity 
(represented by femoral osteonecrosis, small bowel perforation or neurogenic bladder dysfunctions) was reported $^{29}$.

\section{Conclusions}

Anal cancer represents another digestive malignancy in which the sentinel node biopsy technique seems to play a crucial role in identifying the cases which should be further submitted to adjuvant groin irradiation. Due to the relatively low number of cases diagnosed with this pathology, the method is not as well standardised as in breast cancer or malignant melanoma patients. However, the results reported so far are promising, the higher rates of sentinel node detection being reported if a dual method of detection is performed.

\section{Compliance with Ethics Requirements:}

„The authors declare no conflict of interest regarding this article"

\section{References}

1. Daling JR, Madeleine MM, Johnson LG, et al. Human papillomavirus, smoking, and sexual practices in the etiology of anal cancer. Cancer. 2004;101(2):270-280.

2. Siegel RL, Miller KD, Jemal A. Cancer Statistics, 2017. CA Cancer J Clin. 2017; 67(1):7-30.

3. Gerard JP, Chapet O, Samiei F, et al. Management of inguinal lymph node metastases in patients with carcinoma of the anal canal: experience in a series of 270 patients treated in Lyon and review of the literature. Cancer. 2001;92(1):77-84.

4. Bartelink H, Roelofsen F, Eschwege F, et al. Concomitant radiotherapy and chemotherapy is superior to radiotherapy alone in the treatment of locally advanced anal cancer: results of a phase III randomized trial of the European Organization for Research and Treatment of Cancer Radiotherapy and Gastrointestinal Cooperative Groups. J Clin Oncol. 1997;15(5):2040-2049.

5. Socea B, Nica AA, Bratu O, et al. Incidental finding of a sigmoid intussusception associated with rectal prolapse - a case report. Arch Balk Med Union. 2018;53(1):143-146.

6. Bratu OG, Marcu RD, Socea B, et al. Immunohistochemistry particularities of retroperitoneal tumors. Rev Chim (Bucharest). 2018;69(7):1813-1816.

7. Deans GT, McAleer JJ, Spence RA. Malignant anal tumours. Br J Surg. 1994; 81(4):500-508.

8. Oliver GC, Labow SB. Neoplasms of the anus. Surg Clin North Am. 1994; 74(6):1475-1490.

9. Golden GT, Horsley JS, III. Surgical management of epidermoid carcinoma of the anus. Am J Surg. 1976;131(3):275-280.

10. Ryan DE, Taussig D, Steinfeld I et al. Improving CRISPR-Cas specificity with chemical modifications in single-guide RNAs. Nucleic Acids Res. 2018;46(2):792-803.

11. Fuchshuber PR, Rodriguez-Bigas M, Weber T, Petrelli NJ. Anal canal and perianal epidermoid cancers. J Am Coll Surg. 1997;185(5):494-505.
12. Nigro ND, Vaitkevicius VK, Considine B, Jr. Combined therapy for cancer of the anal canal: a preliminary report. Dis Colon Rectum. 1974;17(3):354-356.

13. Myerson RJ, Kong F, Birnbaum EH et al. Radiation therapy for epidermoid carcinoma of the anal canal, clinical and treatment factors associated with outcome. Radiother Oncol. 2001;61(1):15-22.

14. Roelofsen F, Bartelink H. Combined modality treatment of anal carcinoma. Oncologist. 1998;3(6):413-418.

15. Spratt JS. Cancer of the anus. J Surg Oncol. 2000;74(2):173-174.

16. Damin DC, Rosito MA, Gus P, et al. Sentinel lymph node procedure in patients with epidermoid carcinoma of the anal canal: early experience. Dis Colon Rectum. 2003; 46(8):1032-1037.

17. Peley G, Farkas E, Sinkovics I et al. Inguinal sentinel lymph node biopsy for staging anal cancer. Scand J Surg. 2002;91(4):336-338.

18. Bobin JY, Gerard JP, Chapet O, Romestaing P, Isaac S. Lymphatic mapping and inguinal sentinel lymph node biopsy in anal canal cancers to avoid prophylactic inguinal irradiation. Cancer Radiother. 2003;7 Suppl 1:85s-90s.

19. Villa G, Agnese G, Bianchi P, et al. Mapping the sentinel lymph node in malignant melanoma by blue dye, lymphoscintigraphy and intraoperative gamma probe. Tumori. 2000;86(4):343-345.

20. Martin RC, Edwards MJ, Wong SL, et al. Practical guidelines for optimal gamma probe detection of sentinel lymph nodes in breast cancer: results of a multi-institutional study. For the University of Louisville Breast Cancer Study Group. Surgery. 2000;128(2):139-144.

21. Schwartz GF, Guiliano AE, Veronesi U. Proceeding of the consensus conference of the role of sentinel lymph node biopsy in carcinoma or the breast April 19-22, 2001, Philadelphia, PA, USA. Breast J. 2002;8(3):124-138.

22. Pallag A, Rosca E, Tit DM, Mutiu G, Bungau SG, Pop OL. Monitoring the effects of treatment in colon cancer cells using immunohistochemical and histoenzymatic techniques. Romanian Journal of Morphology and Embriology 2015;56(3):1103-1109.

23. Ginghina $\mathrm{O}$, Negrei C, Hudita A, et al. In vitro impact of some natural compounds on HT-29 colorectal adenocarcinoma cells. Farmacia 2017;65(6):947-953.

24. Marcu RD, Spînu AD, Socea B, et al. Castleman's disease clinical, histological and therapeutic features. Rev Chim (Bucharest). 2018;69(4): 823-830.

25. Noorani A, Rabey N, Durrani A, Walsh SR, Davies RJ. Systematic review of sentinel lymph node biopsy in anal squamous cell carcinoma. Int J Surg. 2013;11(9):762-766.

26. Mistrangelo DM, Bello M, Cassoni P, et al. Value of staging squamous cell carcinoma of the anal margin and canal using the sentinel lymph node procedure: an update of the series and a review of the literature. Br J Cancer. 2013;108(3):527-532.

27. Damin DC, Rosito MA, Schwartsmann G. Sentinel lymph node in carcinoma of the anal canal: a review. Eur J Surg Oncol. 2006;32(3):247-252.

28. Mistrangelo M, Morino M. Sentinel lymph node biopsy in anal cancer: a review. Gastroenterol Clin Biol. 2009;33(5):446-450.

29. De Nardi P, Carvello M, Staudacher C. New approach to anal cancer: individualized therapy based on sentinel lymph node biopsy. World J Gastroenterol. 2012; 18(44):6349-6356. 